http://jmscr.igmpublication.org/home/ ISSN (e)-2347-176x ISSN (p) 2455-0450

crossref DOI: https://dx.doi.org/10.18535/jmscr/v8i3.18

\title{
Efficacy of prophylactic retention suture in prevention of wound dehiscence in emergency exploratory laparotomy- A prospective study
}

\author{
Authors \\ Dr Dharmendra Kumar ${ }^{1}$, Dr Raghupathi $S^{2}$,Dr Mohan Kumar $K^{3}$ \\ Dr Asadulla Baig ${ }^{4}$ \\ Department of General Surgery, SDUMC, Tamaka, Kolar, Karnataka, India
}

\begin{abstract}
Introduction: Exploratory laparotomy is routinely performed emergency surgery. Wound dehiscence is the most serious postoperative complications associated with high morbidity and mortality with incidence of 0.4\%-3.5\% and mortality of 10\%-45\%. By transmitting closing forces at the edge of fascia to the different abdominal layers using non-absorbable suture incidence of wound dehiscence can be reduced and retention sutures is one of the recommended technique for same. The aim of this study was to assess the reduced rate of dehiscence in midline laparotomy using prophylactic retention sutures in high-risk patients.

\section{Objectives}

1) To perform normal wound closure in control group.

2) To perform prophylactic retention suture in intervention group.

3) To compare efficacy of the retention suture in prevention of wound dehiscence.

Methods: 50 patients who had undergone emergency exploratory laparotomy in the Department of General Surgery between the study period of October 2018 and September 2019 were included. Patients were randomly divided into two groups- Group A (Interventional Group) and Group B (control Group).

Results: WD occurred in 10 patients (40\%) in the intervention group and 12 control patients (48\%). There was no significant difference in wound infection between the two groups. Post operative pain based on $V A S$ score on POD was found to be 5 in majority of the patients with mean SF6 88.6 and mean hospital stay of 13.2 days of in the intervention group which was 3, mean SF 36 of 92.3 and hospital stay of 10.6 days in control group.

Conclusion: Prophylactic retention sutures reduce the occurrence of WD following midline laparotomy in high-risk patients with multiple risk factors for impaired wound healing.

Keywords: Emergency exploratory laparotomy, Wound dehiscence, Retention suture.
\end{abstract}

\section{Introduction}

Abdominal wound dehiscence or burst abdomen is one of the most serious postoperative complications which is associated with high morbidity and mortality. Its incidence is around $0.4 \%-3.5 \%$ after major abdominal surgeries with a related mortality of around $10 \%-45 \%{ }^{1}$.
The important preoperative risks factors for wound dehiscence are poor nutritional status (clinical cachexia or hypoalbuminemia), emergency surgery, intra-abdominal infection, uncured extensive-stage malignancy, use of corticosteroids in the last 12 months $(>10 \mathrm{mg} / \mathrm{d}$ prednisolone or equivalent for 3 months), uraemia, 
hemodynamic instability $(\mathrm{BP}<90 \mathrm{mmHg})$, haemoglobin $<10 \mathrm{mg} / \mathrm{dL}$ (due to perioperative blood loss or anaemia), predicted abdominal distension (due to ascites or prolonged ileus), chronic pulmonary diseases, clinical jaundice (total bilirubin $>3 \mathrm{mg} / \mathrm{dL}$ ), diabetes mellitus, and age $>60 \mathrm{yr}^{[2-4]}$.

By transmitting closing forces at the edge of fascia to the different abdominal layers using nonabsorbable suture incidence of wound dehiscence can be reduced and retention sutures is one of the recommended technique for reducing disruption of fascia in vulnerable cases ${ }^{5}$.

The purpose of this study is to assess the effect of prophylactic retention sutures in patients who undergo emergency exploratory laparotomy for wound dehiscence.

\section{Objectives}

4) To perform normal wound closure following exploratory laparotomy in control group.

5) To perform prophylactic retention suture following exploratory laparotomy in intervention group.

6) To compare efficacy of the retention suture in prevention of wound dehiscence.

\section{Materials and Methods}

All patients who undergo emergency exploratory laparotomy in the Department of General Surgery of R.L. JALAPPA Hospital between the study period of October 2018 and September 2019.

Sample Size: 50, 25 in each group

Duration of study: 1 year

\section{Inclusion Criteria}

1) Age > 18 years.

2) Patient who undergo emergency exploratory laparotomy through midline incision.

\section{Exclusion Criteria}

1) Patient who have already undergone exploratory laparotomy through midline incision

2) Patients with incisional hernia.

\section{Methods of collection of data}

Patients who are planned for exploratory laparotomy are randomly divided into two groupsGroup A (Interventional Group) and Group B (control Group).

In the control group, the fascia was sutured continuously using a running \#1 nylon suture located $1 \mathrm{~cm}$ from the edge of the linea alba with $1-\mathrm{cm}$ intervals. The running suture was locked intermittently every $5 \mathrm{~cm}$ to divide the long continuous suture into multiple smaller sections. Subcutaneous tissue was sutured using \#2-0 vicryl, and skin was closed using mattress suture of \#2-0 nylon.

In the intervention group, the fascia was sutured using the same technique as the control group; however, retention sutures were added using a \#1 nylon string every $10 \mathrm{~cm}$ and contained $5 \mathrm{~cm}$ of the skin, subcutaneous tissue, rectus muscle, and abdominal fascia (except peritoneum) on each side. The first retention suture was placed $5 \mathrm{~cm}$ above the lower end of the incision and repeated every $10 \mathrm{~cm}$ toward the upper part of the incision. Occurrence of WD (the primary outcome) was assessed daily by precise examination of the wound. Other postoperative outcomes, which were assessed for each group, included evisceration, need to reoperate due to WD, wound infection (based on clinical findings approved by microbiological culture), postoperative pain, length of postoperative hospital stay and postdehiscence in-hospital mortality.

\section{Results}

Data was entered into Microsoft excel data sheet and was analysed using SPSS 22 version software. Categorical data was represented in the form of Frequencies and proportions. Continuous data was represented as mean and SD. ANOVA (Analysis of Variance) or Kruskal Wallis test was the test of significance to identify the mean difference between more than two groups for quantitative and qualitative data respectively.

Age Distribution: Most common age group involved in the intervention group was 41-50 


\section{JMSCR Vol||08||Issue||03||Page 109-116||March}

years but in the control group was 18-30 years with youngest being 18 year and the elder one being 71 year. From the table 1 we observed that most of the patient in the intervention group was middle age group where chances of the wound dehiscence was expected more.

Table 1: Age Distribution

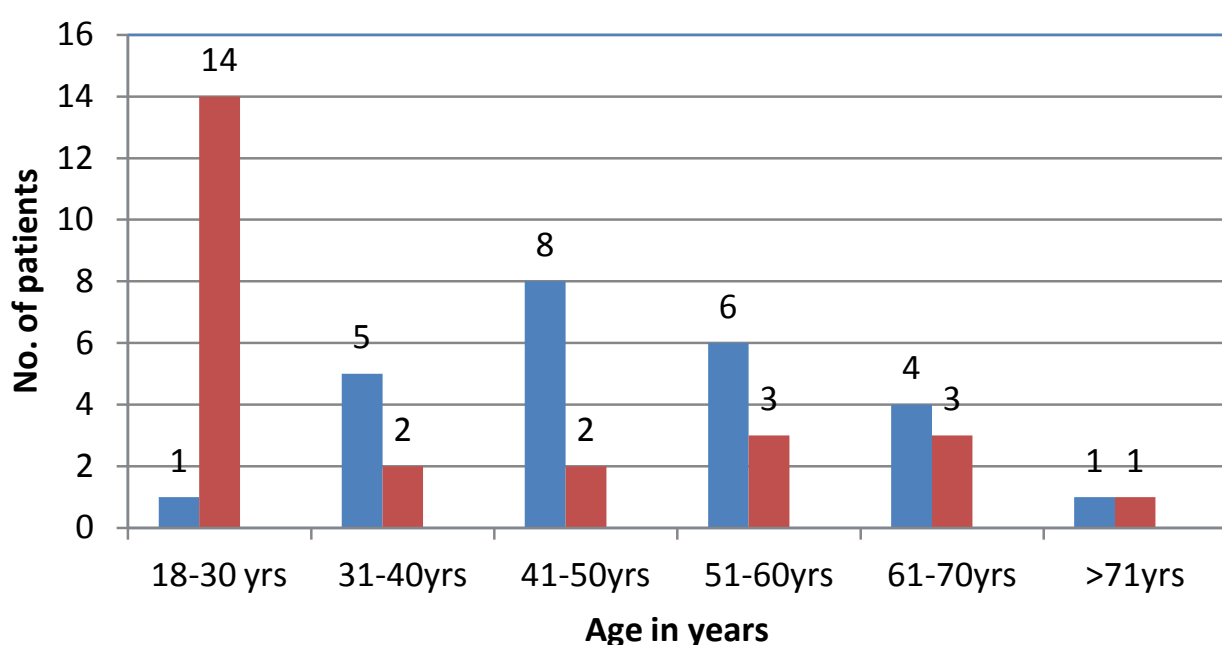

Age in years

Group A

Group B

Column1

Gender Distribution:-Most commonly gender in the study was male in both interventional group and control group as shown in the table no.2

Table No.2: Gender Distribution.

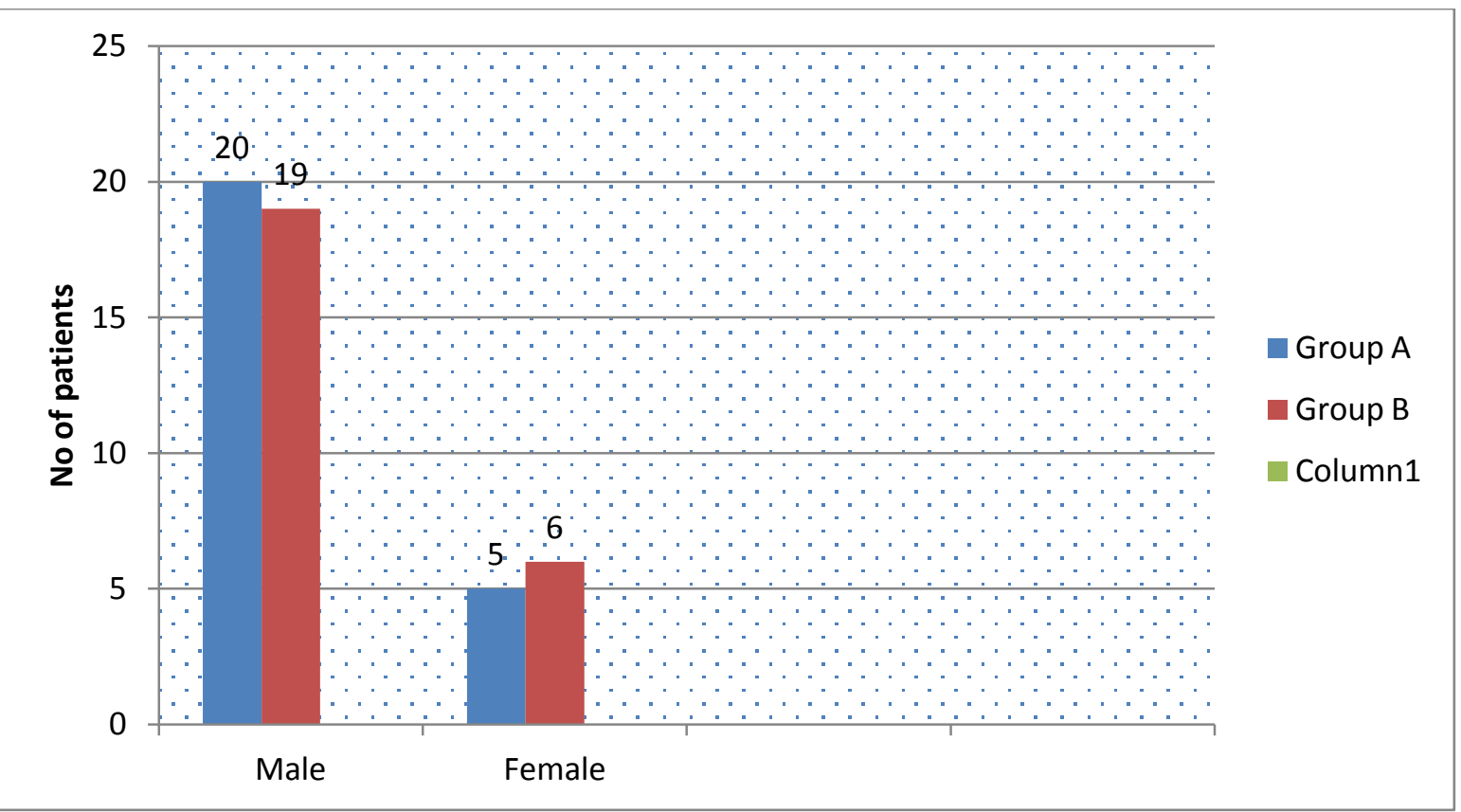

Indication for surgery distribution: As this study includes only emergency exploratory laparotomy hence most common indications for laparotomy for both the group was found to be hollow viscus injury followed by intestinal obstruction in intervention group and blunt trauma 


\section{JMSCR Vol||08||Issue||03||Page 109-116||March}

abdomen in the control group. Among hollow viscus most common viscus perforation was first part of duodenum perforation as shown in the table no. 5 for both the group and the most common procedure performed was Modified Grahm's omental patch repair as shown in the table no. 4. This shows the contamination of the wound in both group was matched.

Table.3: Indications for surgery distribution

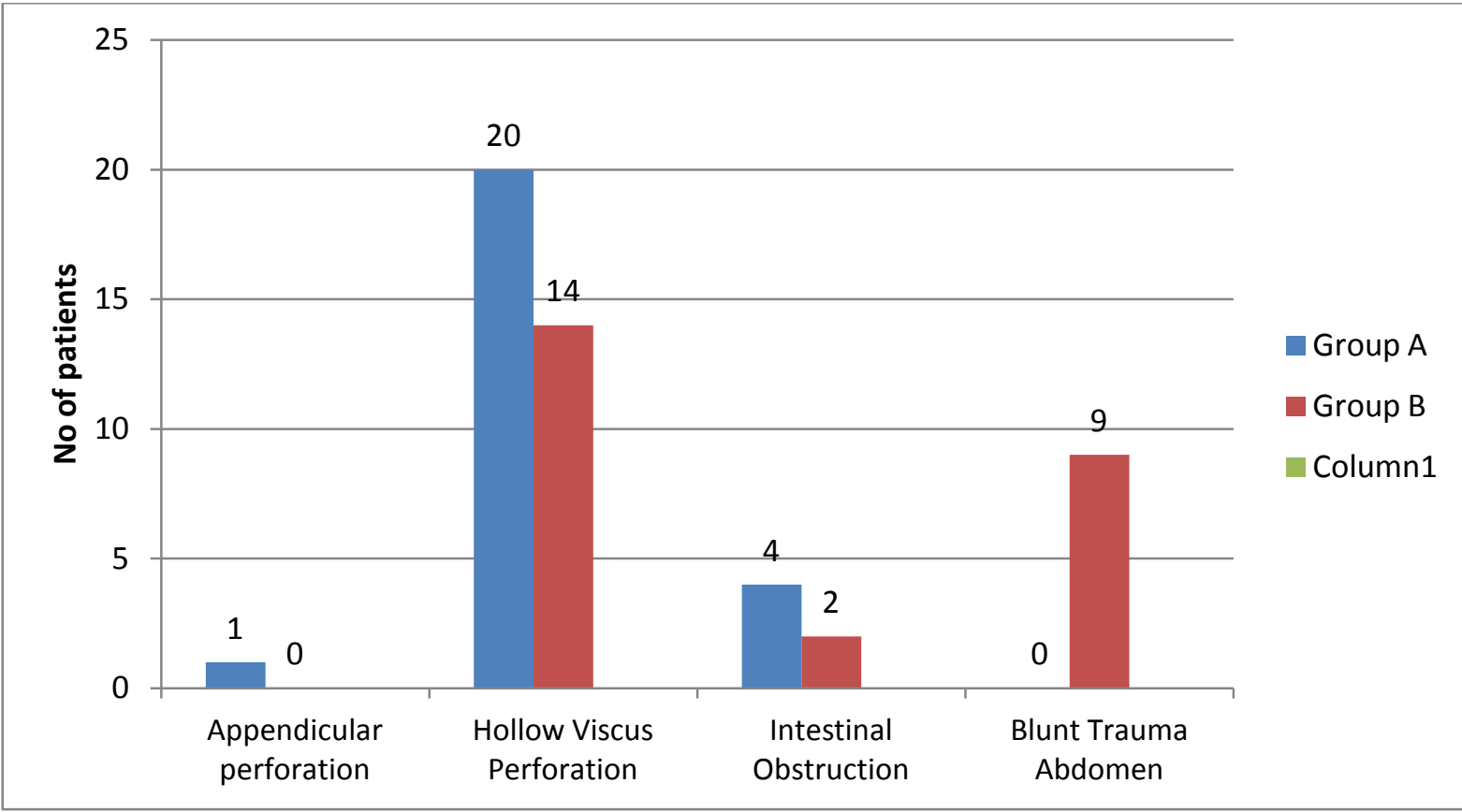

Table No. 4: Treatment Distribution

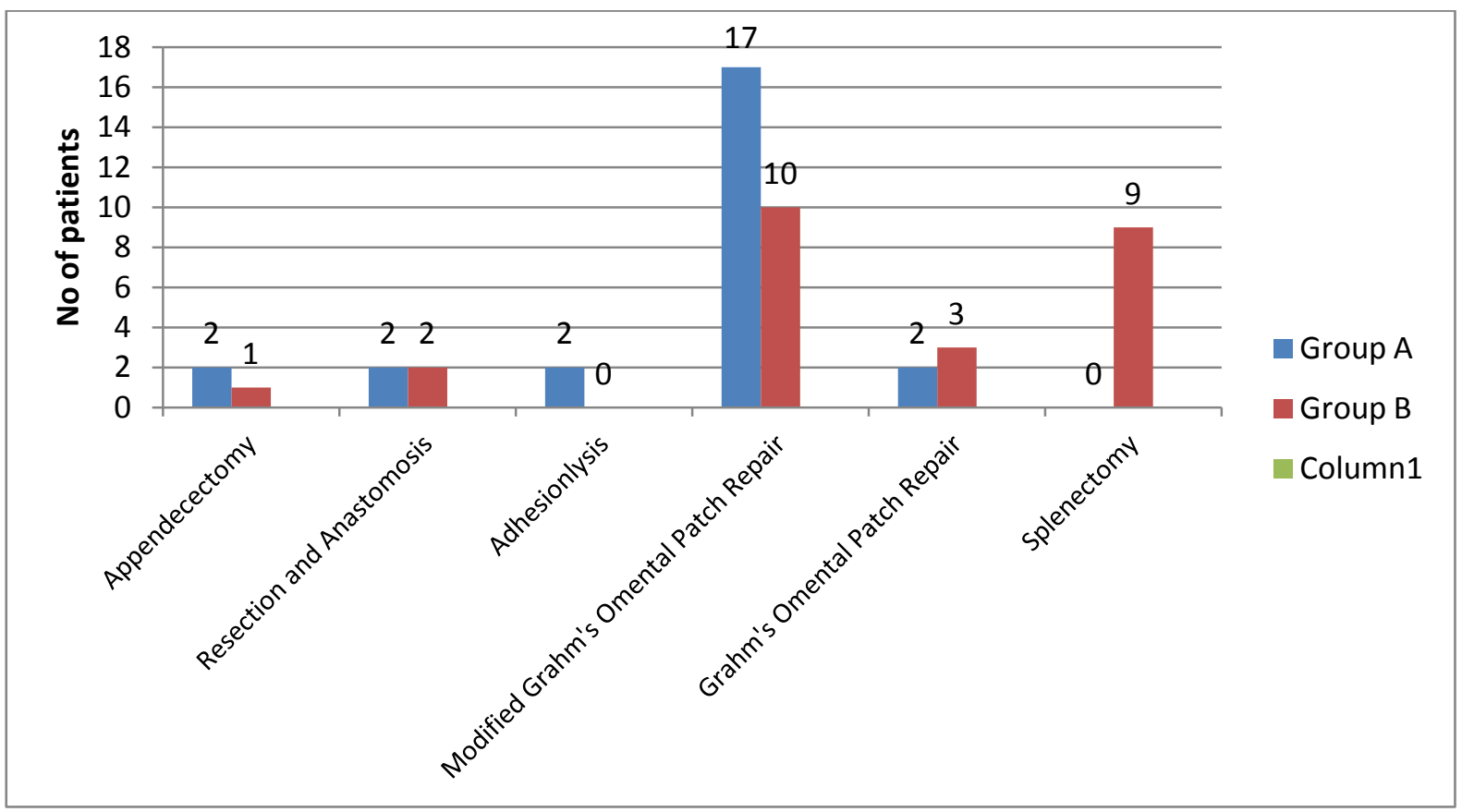


Table No.5: Intra Op findings

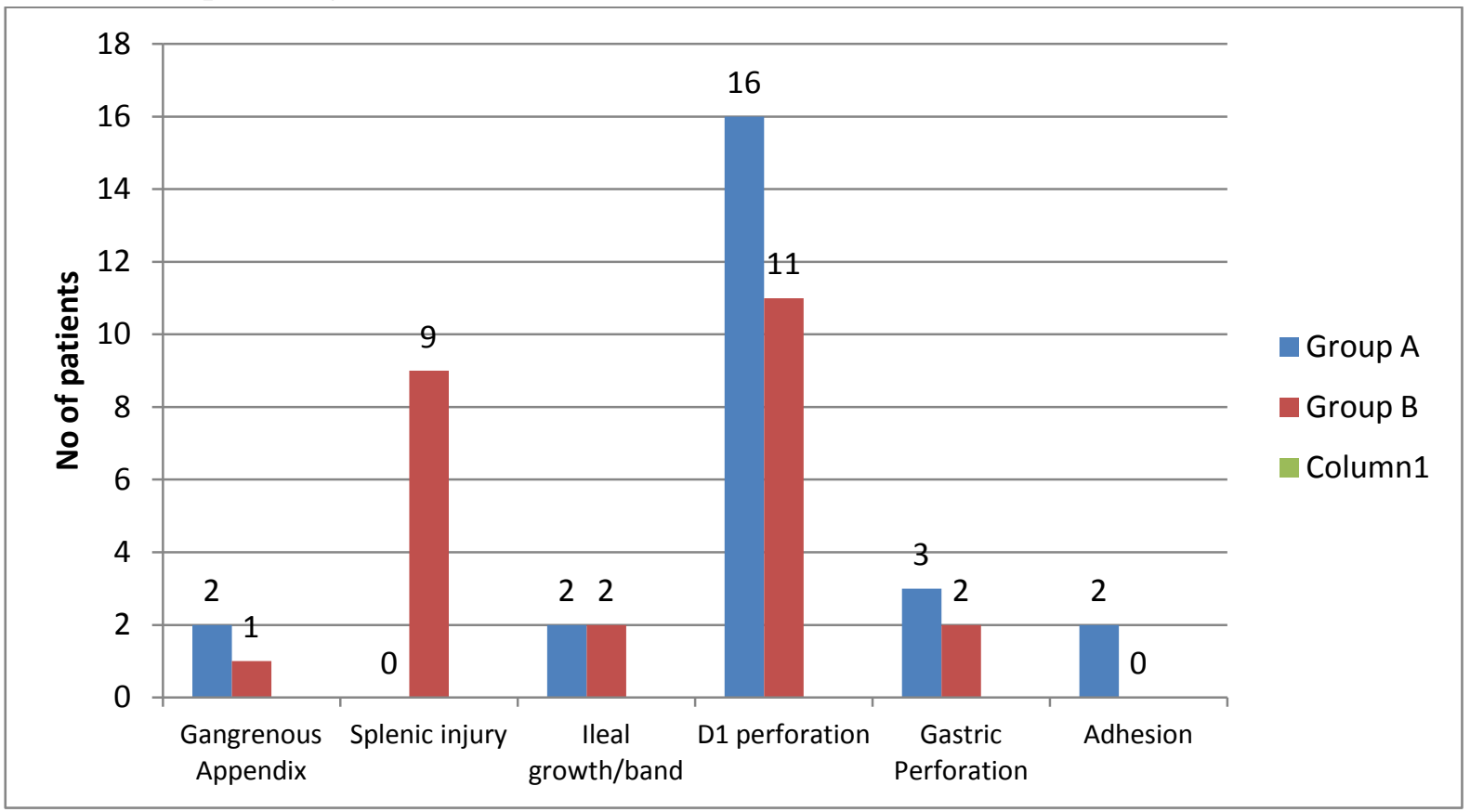

Wound Discharge Distribution: 12 out of 25 patients had wound discharge in the intervention group whereas 10 of 25 patients had wound discharge in the control group as shown in the table no. 6.10 of 12 patients had serous discharge and rest had seropurulent discharge in the intervention group. In the control group, 6 patients had serous discharge and rest had seropurulent.

Table No.6: Wound discharge distribution.

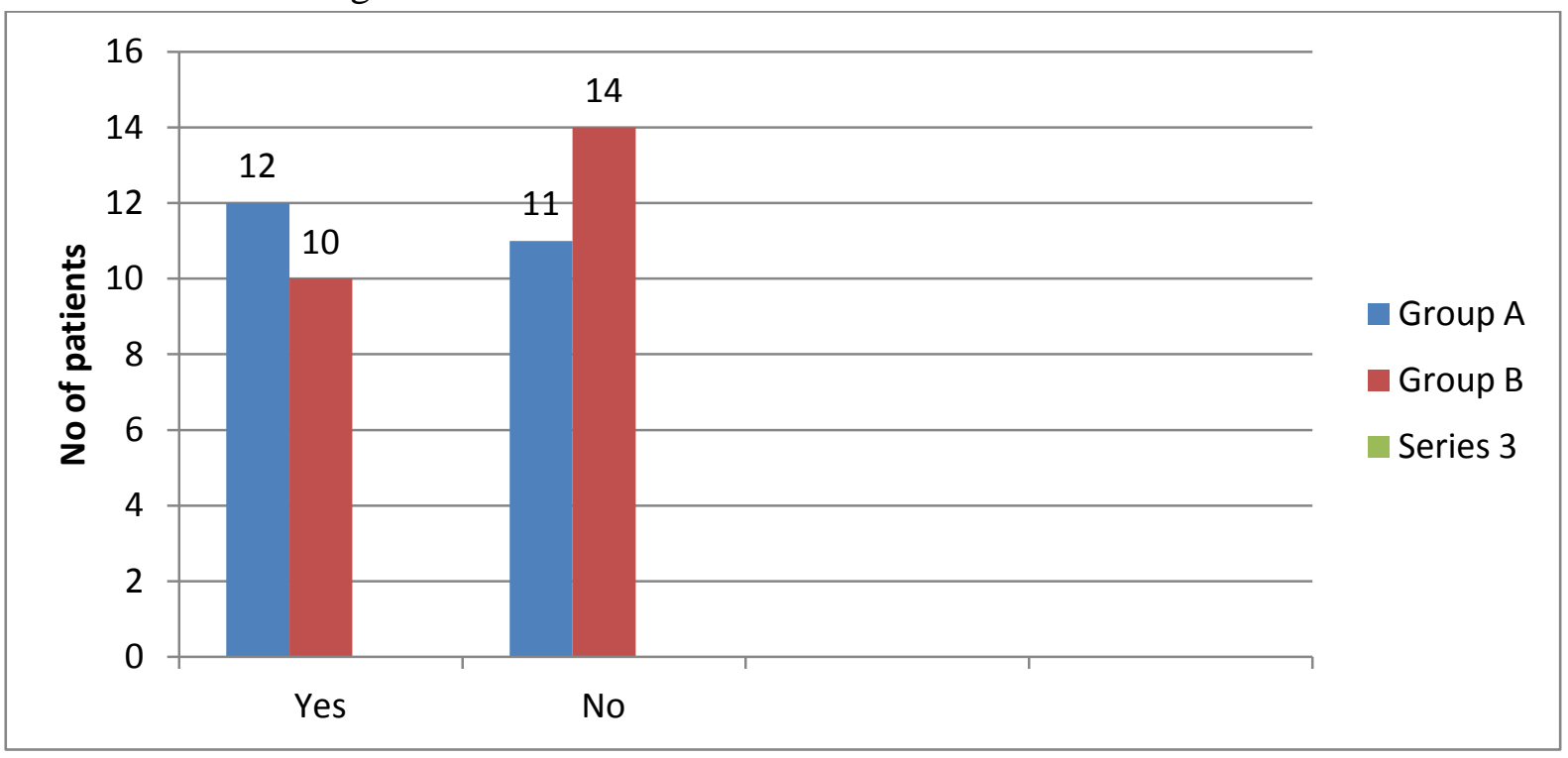

VAS score on POD3 distribution: VAS score assessed on POD for both group and it was found that $92 \%$ had VAS score of 4 or 5 with more than $50 \%$ had VAS score of 5 which showed more pain in the intervention group. 11 of 25 patients had
VAS score of 2 in the control group. Only 2 patients in the group B had VAS score of 6 and the reason being these patient had wound dehiscence. 


\section{JMSCR VoI||08||Issue||03||Page 109-116||March}

Table No.7: VAS distribution

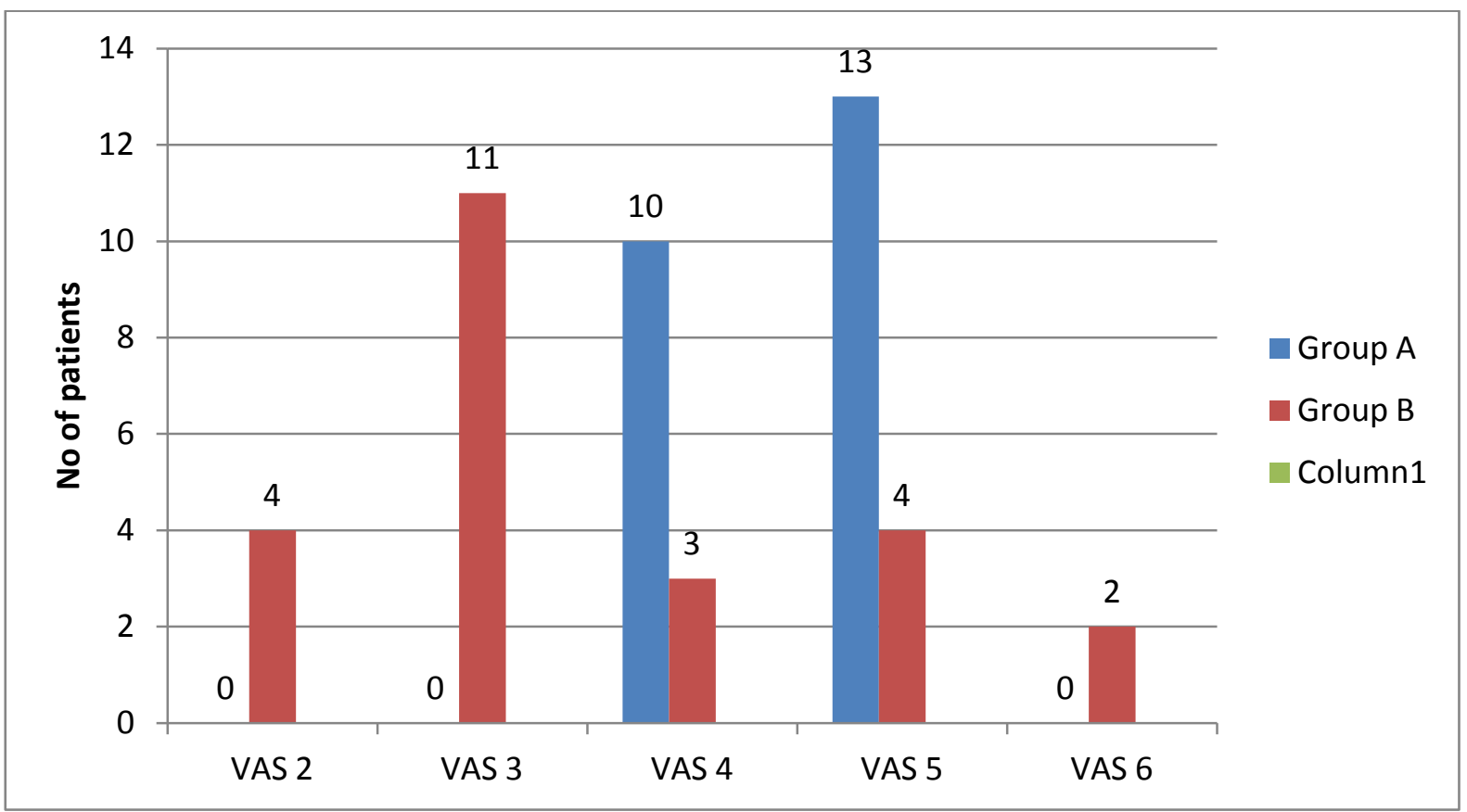

Hospital Stay Distribution: Average hospital stay for the intervention group was found to be 10-15 days comprising around $72 \%$ of the patients with mean hospital stay of 13.2 days whereas in the control group its distributed between $<10$ days and 10-15 days comprising of $76 \%$ of patients with mean hospital stay was 10.6 days. 5 patients in the control group had hospital stay of more than 15 days as shown in table no. 8 and the reason was regular dressing for the wound dehiscence.

Table No.8: Hospital stay distribution

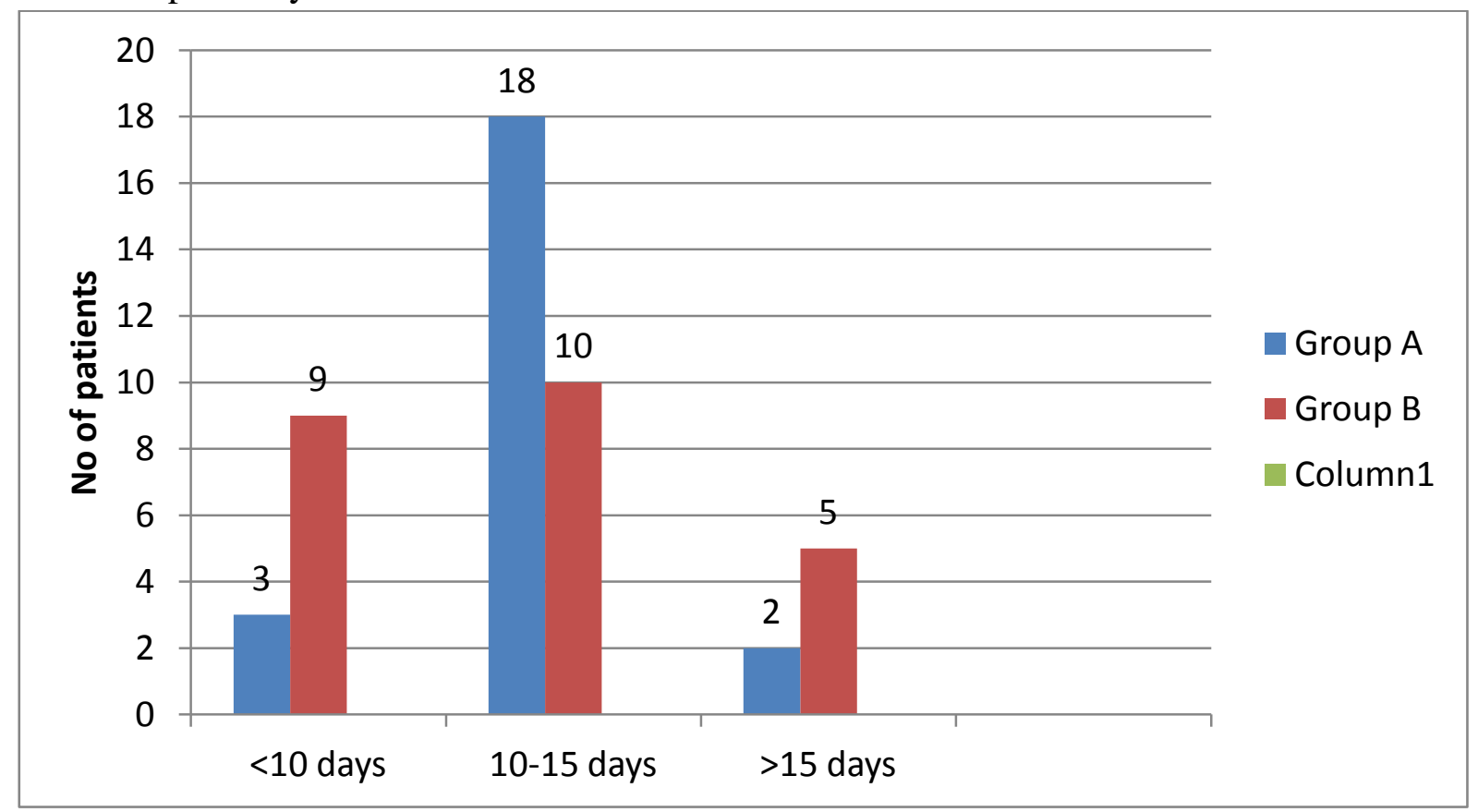

SF 36 Distribution: Mean SF 36 score in the intervention group was around 88.6 whereas in the control group was 92.3 as shown in the table no.9.
This change in the data is attributable to the retention suture causing discomfort to the patients. 


\section{JMSCR Vol||08||Issue||03||Page 109-116||March}

Table No.9: SF 36 distribution.

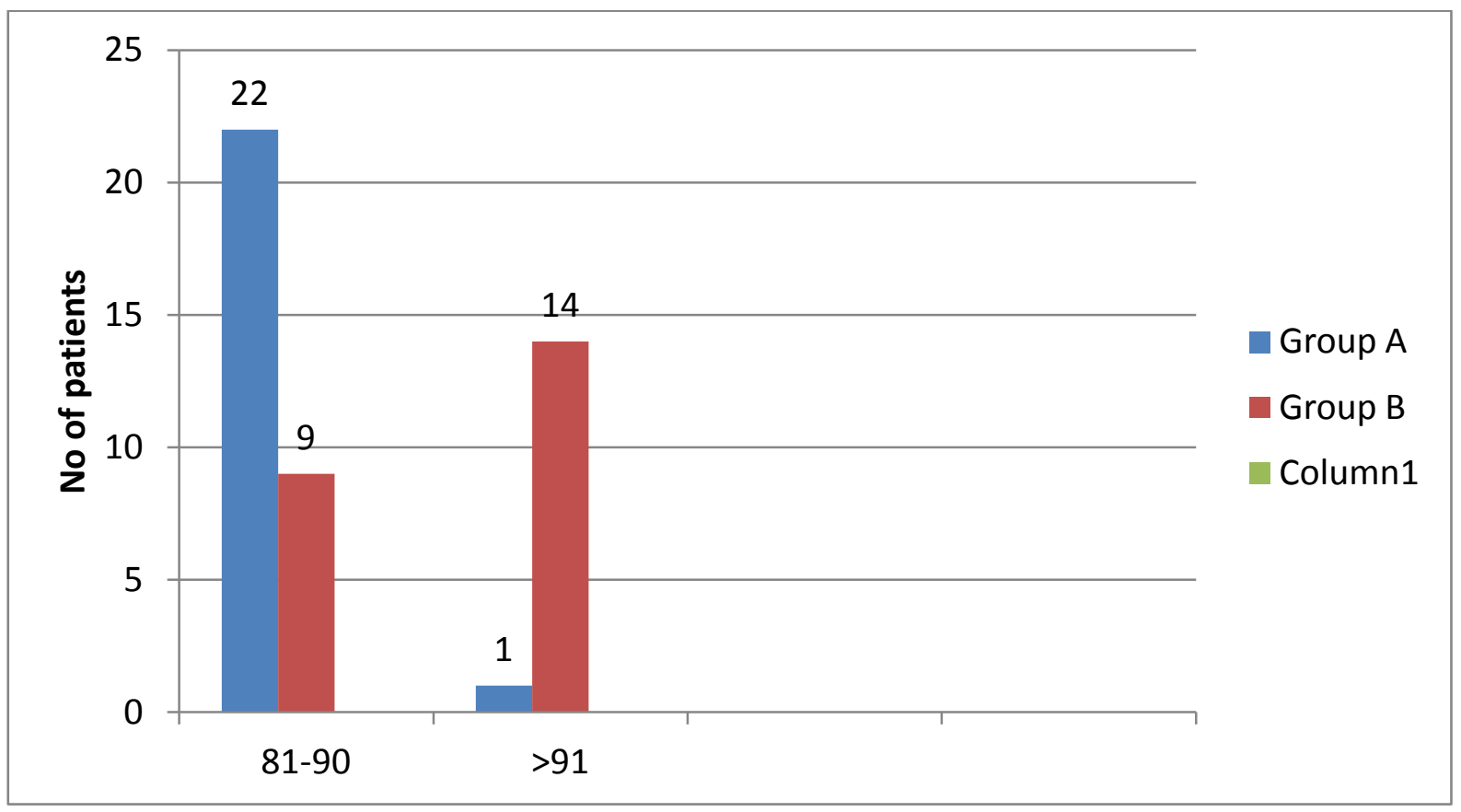

Mortality Distribution: Two patients succumbed in the interventional group as these two patients had 3 days old perorations and patients were in septic shock with AKI. 1 patient in the control group expired and the reason was again the same that is late presentation. This patient had blunt trauma to abdomen and presented one week. The post op image of retention suture group and control group with wound dehiscence is shown in Fig 10.

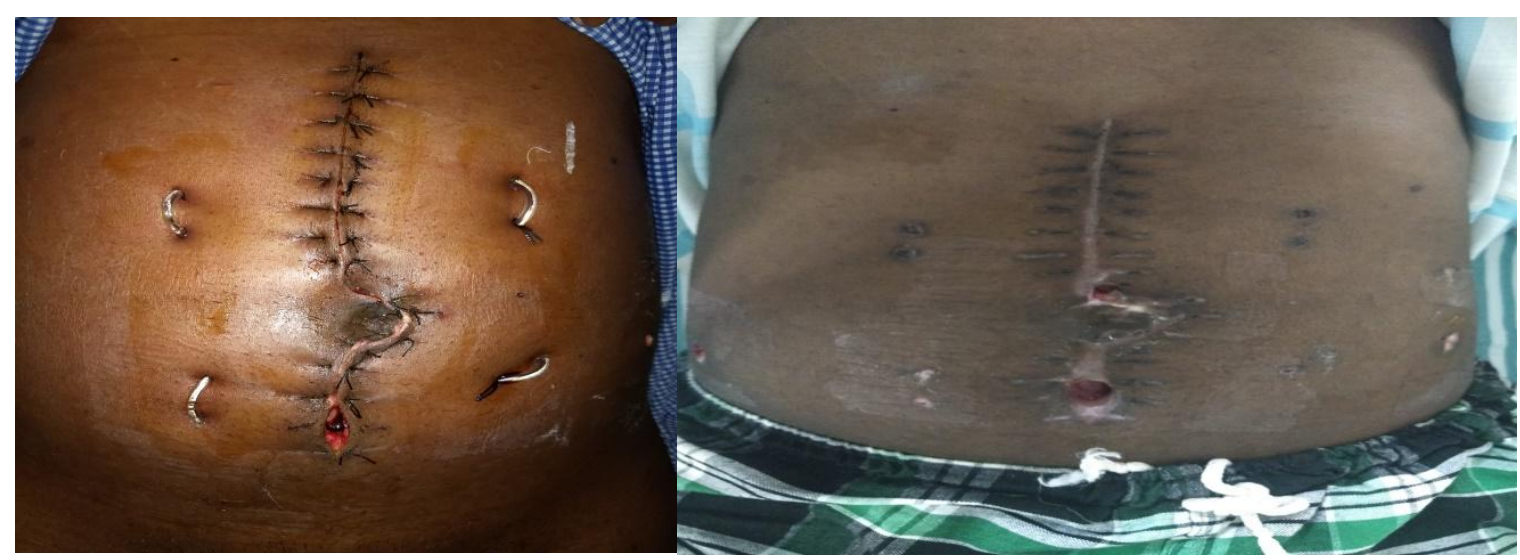

Fig 10a: Retention suture

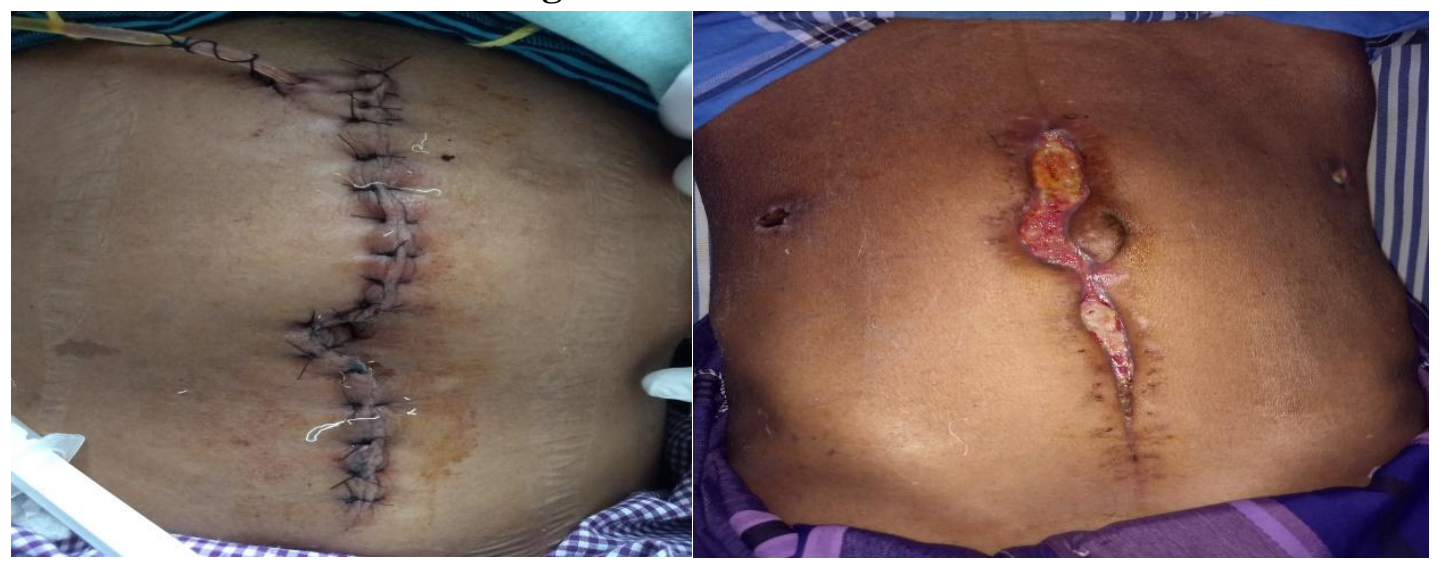

Fig N0 10b: Conventional suture 


\section{Discussion}

- Wound Dehiscence is a devastating incident that can cause pain, mental distress, infectious complications, and financial burdens for the patient, as well as complications including evisceration and reoperation.

- There are several risk factors associated with wound dehiscence and emergency laparotomy is one of the risk factor.

- In the present study it has been observed the presence of 3 or more than 3 risk factors were associated with wound dehiscence which is consistent with Khorgami et al.

- Mean VAS score on POD 3 was around 4.5 which is consistent with Khorgami et al.

- Mean hospital stay in the present study was around 15.8 days which is not in consistent with Khorgami where hospital stay was around 20.4 days.

- Long term complications were not assessed in the present study and the reason being shorter duration of the study and majority of the patient were lost to follow up.

- Mortality in the interventional group was $8 \%$ and $4 \%$ in the control group which was lower in Khorgami et al where $4 \%$ was the mortality in the interventional group.

\section{Conclusion}

- We observed that prophylactic retention sutures could reduce wound dehiscence in midline laparotomy in high-risk patients with multiple risk factors for wound dehiscence without imposing remarkable postoperative complications.

\section{References}

1. Zhamak K, Saeed S, Bardia L, Ali A, Negin H A, Ahmadreza S. Prophylactic retention sutures in midline laparotomy in high-risk patients for wound dehiscence: A randomized controlled trial. J Surg Res 2012: E1-E6.

2. Mohanad A. Effect Of Retention Sutures For Prevention Of Abdominal Wound Dehiscence After Laparotomy In High Risk Patients(A Prospective Study). IOSR J Pharm 2014;4: 38-43.

3. Van Ramshorst GH, Nieuwenhuizen J, Hop WCJ, et al. Abdominal wound dehiscence in adults: Development and validation of a risk model. World J Surg 2010;34:20.

4. Afzal S, Bashir MM. Determinants of wound dehiscence in abdominal surgery in public sector hospital. Annals of King Edward Medical University 2008;14:110.

5. Cigdem MK, Onen A, Otcu S, et al. Postoperative abdominal evisceration in children: Possible risk factors. PediatrSurgInt 2006;22:677.

6. Sørensen LT, Hemmingsen U, Kallehave $\mathrm{F}$, et al. Risk factors for tissue and wound complications in gastrointestinal surgery. Ann Surg 2005;241:654.

Conflict of interest: None 